\title{
Comparison of cause of death coding on death certificates with coding in the Royal College of General Practitioners Oral Contraception Study
}

\author{
SALLY J. WINGRAVE \\ From the Royal College of General Practitioners, Manchester Research Unit \\ VALERIE BERAL \\ From the London School of Hygiene and Tropical Medicine
}

\author{
A. M. ADELSTEIN \\ From the Office of Population Censuses and Surveys, London
}

C. R. KAY

From the Royal College of General Practitioners, Manchester Research Unit

SUMmaRY A comparison has been made between the coding of the cause of death by (a) the Royal College of General Practitioners (RCGP) during the Oral Contraception Study and (b) the Office of Population Censuses and Surveys (OPCS) or the General Register Office for Scotland (GRO) on death certificates for the same subjects. Broad groupings of the International Classification of Diseases (ICD) showed close agreement between RCGP and OPCS or GRO coding for all deaths which occurred from the start of the Oral Contraception Study in 1968 up to December 1978. Moreover, where discrepancies occurred there were no systematic differences between ever-users of oral contraceptives and non-users. Detailed examination of discrepancies in the coding of the causes of those deaths included in the RCGP publication of October $1977^{1}$ shows that our previous estimate of mortality risk associated with oral contraceptives would not be materially altered by the use of death certificate information.

In October 1977, the Royal College of General Practitioners published analyses of the distribution of deaths recorded in its ongoing, long-term, prospective Oral Contraception Study, which began in $1968 .{ }^{1}$ It was noted that the total mortality rate in women who had ever used the pill was increased by $40 \%$ and was accounted for by an increase in deaths from circulatory diseases of one in 5000 ever-users a year. The cause of death used in the analysis was that reported by the participating general practitioners (GPs) and was coded by C.R.K. and subsequently checked by V.B. Because of the importance of these findings, it was felt necessary to investigate the extent to which the conclusions might be altered if information from death certificates was used instead. Since the analysis of October 1977, additional deaths have been reported in the Oral Contraception Study, and these provide an opportunity to make a general comparison of the cause of death reported by the GP with that supplied and coded by the OPCS or the GRO, referred to hereafter as the registries.

\section{Method}

The design of the Oral Contraception Study has been described in detail elsewhere. ${ }^{2}$ Briefly, 23000 pill takers and 23000 controls, who were matched for age and marital status, were recruited over a 14-month period in 1968-69. Controls who later became oral contraceptive users were included in the taker category from the time of change. Ex-takers who resumed the use of oral contraceptives were, 
thereafter, excluded from the analyses published so far. At six-monthly intervals each GP reports on the occurrence of illness, pregnancy or death, and on the details of oral contraceptive use in the study population.

For all deaths the GPs are asked to report the causes in the same format as required for the death certificate. The underlying cause of death is assigned by C.R.K. based upon the description given by each GP, supplemented by information relating to previous medical history gathered during the study. This cause of death is then coded using the International Classification of Diseases (ICD) 8th revision. ${ }^{3}$ Once coded, the information is stored on computer. Before analyses of mortality are begun, each code is checked by an independent observer. For the purposes of the present comparison, this second stage of checking has not been undertaken for those deaths not included in the analyses published in October 1977, since this could not have been done without being influenced by the information on copies of the respective death certificates which are now available.

Copies of the death certificates were obtained by applying to the GPs who, in order to maintain confidentiality, forwarded the name and address at death of relevant patients either to the OPCS in London or to the GRO in Edinburgh. By arrangement with these offices, personal details of the patients were blanked out on the photocopies of the death certificates sent to the RCGP. Because of the small number of subjects in Northern Ireland, it was decided not to involve the separate National Health Service Register maintained there.

Information on a death certificate relating to the cause of death is supplied by either a coroner or a doctor, who may be the patient's own GP or another medical practitioner attendant at death, such as a hospital doctor. Subsequent assignment and coding of the 'underlying cause of death' at the registries is performed by trained staff. The concept of an 'underlying cause of death' is that formulated by the World Health Organisation, and instructions about the assignment of underlying cause are included in the $I C D$ manual. ${ }^{3}$

The ICD includes about 1000 three-digit categories. These are aggregated into various lists, for example the ' $A$ ' and ' $B$ ' lists with 150 and 50 categories respectively. In this paper the $I C D$ codings of deaths available since the start of the Oral Contraception Study have been compared at the three-digit, the ' $A$ ' list, and the ' $B$ ' list levels. The ' $B$ ' list classification, which is the most aggregated, generally provides the best agreement. ${ }^{4}$ Since these broad groupings are sufficiently specific for most epidemiological purposes, many of the comparisons presented here are confined to the ' $B$ ' list codings. Codes which are discrepant at the ' $B$ ' list level have been individually investigated to determine possible reasons for the differences. Because of our particular interest in circulatory deaths, more detailed analysis of these has been performed using the ' $A$ ' list codings. Differences between ever-users (takers and ex-takers) and non-users (controls) have also been examined. The deaths discussed in the publication of October 1977 have been analysed separately to determine whether the use of OPCS codings would impair the validity of the original conclusions.

\section{Results}

TOTAL DEATHS FROM START OF ORAL CONTRACEPTION STUDY UP TO DECEMBER 1978 (a) Comparison of all death codes

By December 1978, a total of 249 deaths had been recorded in the study. Death certificates were unobtainable for $\mathbf{4 4}$ of these because GPs did not respond (16 cases), the deaths occurred in Northern Ireland (three cases), or the death certificates could not be traced by the registries ( 25 cases). As shown in Table 1 , of the 205 deaths available for analysis, 171 (83\%) were coded to non-violent causes and 31 (15\%) to violent causes, including suicide, by both the RCGP and the registries; in three cases $(1.5 \%)$ the death was coded as non-violent by the RCGP and violent by the registries. (The details of these are listed in Table 2). Of the 171 non-violent deaths, the three-digit ICD codes were identical in $122(71 \%)$, the ' $A$ ' list codes were identical in $143(84 \%)$, and the ' $B$ ' list in $150(88 \%)$. All the 21 cases (12\%) which were discrepant according to the ' $\mathrm{B}$ ' list are shown in Table 2.

The 31 accidental and violent causes of death coded by both the RCGP and the registries were compared according to the four ' $\mathrm{B}$ ' list categories relating to external causes of injury. Complete coding agreement between the RCGP and the registries

Table 1 Comparison of deaths attributed to violent and non-violent causes by RCGP or OPCS/GRO

\begin{tabular}{llll}
\hline & \multicolumn{3}{c}{ RCGP STUDY } \\
& $\begin{array}{l}\text { Non-violent causes } \\
\text { (ICD 000-796.9) }\end{array}$ & $\begin{array}{l}\text { Violent causes } \\
\text { (ICD E800-E999) }\end{array}$ & Total \\
\hline $\begin{array}{l}\text { OPCS/GRO } \\
\text { Non-violent } \\
\text { causes }\end{array}$ & 0 & 171 \\
$\begin{array}{l}\text { (ICD 000-796.9) } \\
\begin{array}{l}\text { Violent causes } \\
\text { (ICD E800-E999) }\end{array}\end{array}$ & $3^{*}$ & 0 & 34 \\
\hline Total & 174 & 31 & 205 \\
\hline
\end{tabular}

'Patient numbers 15595, 16328, 30279 (see Table 2 for details). 
Table 2 List of the 32 cases with discrepant coding at ' $B$ ' list level (total cases = 205)

\begin{tabular}{|c|c|c|c|c|c|c|c|c|}
\hline $\begin{array}{l}\text { Study } \\
\text { number }\end{array}$ & $\begin{array}{l}\text { Place of } \\
\text { death }\end{array}$ & Cohort & Necropsy & Certifier & $R C G P$ & $I C D$ & OPCS/GRO & $I C D$ \\
\hline 05765 & Home & $\begin{array}{l}\text { Ever- } \\
\text { user }\end{array}$ & Yes & Other & $\begin{array}{l}\text { 1a Suicide, poisoning } \\
\text { (Mandrax) }\end{array}$ & E950 & $\begin{array}{l}\text { 1a Pulmonary haemorrhage } \\
\text { b Prolonged unconsciousness } \\
\text { c Arvynol poison (suicide) }\end{array}$ & E980 \\
\hline 06389 & Hospital & Control & No & GP & $\begin{array}{l}\text { 1a Paroxysmal tachycardia } \\
\text { b Paralytic ileus } \\
\text { c Hysterectomy }\end{array}$ & 560 & $\begin{array}{l}\text { 1a Paroxysmal tachycardia } \\
\text { b Paralytic ileus } \\
\text { c Hysterectomy (fibroids) }\end{array}$ & 218 \\
\hline 06444 & Home & Control & Yes & Other & $\begin{array}{l}\text { 1a Duodenal ulcer, } \\
\text { intestinal obstruction } \\
\text { pyloric stenosis }\end{array}$ & 560 & $\begin{array}{l}\text { 1a Intestinal obstruction } \\
\text { b Adhesion due to chronic } \\
\text { duodenal ulcer }\end{array}$ & 532 \\
\hline${ }^{*} 09477$ & Home & $\begin{array}{l}\text { Ever- } \\
\text { user }\end{array}$ & Yes & Coroner & 1a Suicide (hanging) & E963 & $\begin{array}{l}\text { 1a Asphyxiation by hanging } \\
\text { (suicide) }\end{array}$ & E953 \\
\hline *10695 & Hospital & $\begin{array}{l}\text { Ever- } \\
\text { user }\end{array}$ & Yes & Coroner & $\begin{array}{l}\text { 1a Uraemia } \\
\text { b Chronic renal failure } \\
\text { c Malignant hypertension } \\
\text { (Past history of hypertension } \\
\text { since } 1970 \text { and malignant } \\
\text { hypertension and uraemia } \\
\text { since July 1972) }\end{array}$ & 4003 & $\begin{array}{l}\text { 1a Coronary insufficiency, } \\
\text { Coronary atherosclerosis } \\
\text { Malignant hypertension }\end{array}$ & 4110 \\
\hline *14290 & Hospital & $\begin{array}{l}\text { Ever- } \\
\text { user }\end{array}$ & No & Other & $\begin{array}{l}\text { 1a Peritonitis } \\
\text { b Ischaemic colitis } \\
\text { (GP's letter received } \\
\text { subsequently: 'This patient } \\
\text { died under general } \\
\text { anaesthetic whilst having an } \\
\text { operation to have a fibroid } \\
\text { removed'.) }\end{array}$ & 444 & $\begin{array}{l}\text { 1a Shock } \\
\text { b Faecal peritonitis } \\
\text { c Ovarian cyst }\end{array}$ & 220 \\
\hline$* 15595$ & Unknown & Control & Yes & Coroner & $\begin{array}{l}\text { 1a Renal failure } \\
\text { b Ovarian cancer } \\
\text { (Laparotomy for disseminated } \\
\text { carcinoma of ovary one month } \\
\text { before death) }\end{array}$ & 183 & $\begin{array}{l}\text { 1a Acute hepatorenal failure } \\
\text { b Mercury intoxication }\end{array}$ & E980 \\
\hline$* 15773$ & Hospital & $\begin{array}{l}\text { Ever- } \\
\text { user }\end{array}$ & Yes & Other & $\begin{array}{l}\text { 1a Cirrhosis of liver } \\
\text { and septicaemia } \\
\text { b Chronic cholangitis } \\
\text { c Congenital abnormality of } \\
\text { intrahepatic bile ducts } \\
\text { (Recurrent cholangitis since } \\
\text { 1968) }\end{array}$ & 571 & 1a Caroli's disease & 758 \\
\hline 16328 & Hospital & $\begin{array}{l}\text { Ever- } \\
\text { user }\end{array}$ & Yes & Coroner & $\begin{array}{l}\text { 1a Massive cerebral haemorrhage } \\
\text { Small liver nodules noted } \\
\text { (no mention of fall) }\end{array}$ & 431 & $\begin{array}{l}\text { 1a Cerebral haemorrhage } \\
\text { b Fall at work (open } \\
\text { verdict) }\end{array}$ & E988 \\
\hline 17332 & Hospital & $\begin{array}{l}\text { Ever- } \\
\text { user }\end{array}$ & Yes & Coroner & $\begin{array}{l}\text { 1a Massive gastrointestinal } \\
\text { haemorrhage } \\
\text { b Acute gastric erosion } \\
\text { c Peritonitis following } \\
\text { partial gastrectomy }\end{array}$ & 531 & $\begin{array}{l}\text { 1a Massive gastrointestinal } \\
\text { haemorrhage } \\
\text { b Acute gastric erosion } \\
\text { c Peritonitis }\end{array}$ & 567 \\
\hline 17565 & Hospital & $\begin{array}{l}\text { Ever- } \\
\text { user }\end{array}$ & Yes & Coroner & $\begin{array}{l}\text { 1a Cerebral haemorrhage } \\
\text { b Hypertension } \\
\text { c Chronic nephritis } \\
\text { (Past history of chronic } \\
\text { pyelonephritis and } \\
\text { hypertension) }\end{array}$ & 590 & $\begin{array}{l}\text { 1a Subdural haemorrhage } \\
\text { b Hypertension } \\
\text { c Renal artery stenosis }\end{array}$ & 431 \\
\hline 17910 & Home & $\begin{array}{l}\text { Ever- } \\
\text { user }\end{array}$ & Yes & Coroner & $\begin{array}{l}\text { 1a Bronchial asthma } \\
\text { b Diabetes mellitus } \\
\text { (Past history of diabetes and } \\
\text { asthma. Admitted to hospital } \\
\text { four months before death with } \\
\text { acute asthma) }\end{array}$ & 493 & $\begin{array}{l}\text { 1a Bronchial asthma } \\
\text { b Diabetes mellitus }\end{array}$ & 250 \\
\hline 21534 & Unknown & Control & Yes & Other & $\begin{array}{l}\text { 1a Suicide } \\
\text { b Drug overdose }\end{array}$ & E950 & 1a Barbiturate overdose & E980 \\
\hline *23726 & Hospital & $\begin{array}{l}\text { Ever- } \\
\text { user }\end{array}$ & Yes & Coroner & $\begin{array}{l}\text { 1a Acute cardiac failure } \\
\text { b Paradoxical coronary embolism } \\
\text { c Deep internal iliac thrombosis } \\
\text { d Contraceptive pill, natural } \\
\text { causes }\end{array}$ & 410 & $\begin{array}{l}\text { 1a Acute cardiac failure } \\
\text { b Paradoxical coronary embolism } \\
\text { c Deep internal iliac thrombosis } \\
\text { d Contraceptive pill, natural } \\
\text { causes }\end{array}$ & 453 \\
\hline
\end{tabular}


Table 2 (continued)

\begin{tabular}{|c|c|c|c|c|c|c|c|c|}
\hline $\begin{array}{l}\text { Study } \\
\text { number }\end{array}$ & $\begin{array}{l}\text { Place of } \\
\text { death }\end{array}$ & Cohort & Necropsy & Certifier & $R C G P$ & $I C D$ & OPCS/GRO & $I C D$ \\
\hline 23951 & Hospital & Control & No. & Other & $\begin{array}{l}\text { 1a Rheumatoid disease } \\
\text { b Leucopaenia, pericardial } \\
\text { effusion, collagen disease }\end{array}$ & 734 & $\begin{array}{l}\text { 1a Acute renal failure } \\
\text { II Rheumatoid arthritis }\end{array}$ & 580 \\
\hline 25737 & Home & Control & Yes & Coroner & 1a Suicide (gas poisoning) & E870 & $\begin{array}{l}\text { 1a Carbon monoxide poisoning } \\
\text { (self-administered) }\end{array}$ & E951 \\
\hline 25929 & Hospital & $\begin{array}{l}\text { Ever- } \\
\text { user }\end{array}$ & Yes & Coroner & $\begin{array}{l}\text { 1a Overdose } \\
\text { (History of puerperal depressior } \\
\text { and last child born } 15 \text { months } \\
\text { before death) }\end{array}$ & on & $\begin{array}{l}\text { 1a Poisoning by ingesting } \\
\text { overdose Prothiadene with } \\
\text { insufficient evidence to } \\
\text { show intention }\end{array}$ & E980 \\
\hline *28008 & Hospital & $\begin{array}{l}\text { Ever- } \\
\text { user }\end{array}$ & Yes & Other & 1a Malignant neoplasm brain & 191 & 1a Corpus callosum neoplasm & 238 \\
\hline *30279 & Unknown & Control & No & Other & 1a Natural causes & 796 & $\begin{array}{l}\text { 1a Natural causes } \\
\text { (asphyxia following } \\
\text { inhalation of vomitus) }\end{array}$ & E911 \\
\hline 31657 & Hospital & $\begin{array}{l}\text { Ever- } \\
\text { user }\end{array}$ & No & Other & 1a Disseminated sclerosis & 340 & $\begin{array}{l}\text { 1a Bronchopneumonia followed } \\
\text { by respiratory failure } \\
\text { II Multiple sclerosis }\end{array}$ & 485 \\
\hline 32755 & Unknown & $\begin{array}{l}\text { Ever- } \\
\text { user }\end{array}$ & No & Other & $\begin{array}{l}\text { 1a Overdose paracetamol } \\
\text { (Telephone conversation with } \\
\text { GP revealed suicide) }\end{array}$ & E950 & $\begin{array}{l}\text { 1a Acute hepatocellular } \\
\text { failure } \\
\text { b Paracetamol overdose }\end{array}$ & $\mathrm{E} 850$ \\
\hline *33967 & Unknown & Control & No & Other & Cerebral haemorrhage & 431 & $\begin{array}{l}\text { 1a Cerebral haemorrhage } \\
\text { b Benign hypertension } \\
\text { c Obesity (gross) } \\
\text { II Respiratory failure }\end{array}$ & 277 \\
\hline 35538 & Hospital & $\begin{array}{l}\text { Ever- } \\
\text { user }\end{array}$ & No & Other & 1a Crohn's disease & 563 & $\begin{array}{l}\text { 1a Pulmonary embolism } \\
\text { II Crohn's disease }\end{array}$ & 450 \\
\hline 35587 & Home & Control & Yes & Coroner & $\begin{array}{l}\text { 1a Pneumonia } \\
\text { b Dystrophy myotonica } \\
\text { (Past history of dystrophy } \\
\text { myotonica for three months) }\end{array}$ & 330 & $\begin{array}{l}\text { 1a Congestive cardiac failure } \\
\text { b Chronic bronchitis } \\
\text { II Terminal bilateral broncho- } \\
\text { pneumonia. Natural causes. }\end{array}$ & 491 \\
\hline 36142 & Hospital & Control & No & Other & $\begin{array}{l}\text { 1a Decubitus ulcer } \\
\text { b Septicaemia } \\
\text { c Renal failure } \\
\text { (Past history of hypertension } \\
\text { and congenital deformity of legs. } \\
\text { Sciatica. Coded hypertensive } \\
\text { renal failure) }\end{array}$ & s. & $\begin{array}{l}\text { 1a Chronic sepsis } \\
\text { b Decubitus ulcer } \\
\text { c Osteoarthritis }\end{array}$ & 713 \\
\hline 42101 & Hospital & Control & Yes & Coroner & 1a Head injuries & E929 & $\begin{array}{l}\text { 1a Bronchopneumonia } \\
\text { b Fractured temporal bone } \\
\text { c Knocked down by motor } \\
\text { vehicle when crossing road }\end{array}$ & E814 \\
\hline$* 42479$ & Hospital & Control & Yes & Coroner & $\begin{array}{l}\text { 1a Cerebral haemorrhage } \\
\text { (No past history of renal } \\
\text { disease) }\end{array}$ & 431 & $\begin{array}{l}\text { 1a Cerebral haemorrhage } \\
\text { b Hypertension, cardiovascular } \\
\text { disease } \\
\text { c Chronic pyelonephritis } \\
\text { right kidney }\end{array}$ & 590 \\
\hline 61015 & Hospital & Control & No & Other & $\begin{array}{l}\text { 1a Chronic renal failure } \\
\text { following unsuccessful renal } \\
\text { transplant }\end{array}$ & 582 & $\begin{array}{l}\text { 1a Coronary thrombosis } \\
\text { II Renal failure }\end{array}$ & 410 \\
\hline *61375 & Home & Control & No & Other & $\begin{array}{l}\text { 1a Septicaemia } \\
\text { b Gastroenteritis }\end{array}$ & 038 & $\begin{array}{l}\text { 1a Septicaemia } \\
\text { b Gastroenteritis }\end{array}$ & 009 \\
\hline 62075 & Home & $\begin{array}{l}\text { Ever- } \\
\text { user }\end{array}$ & Yes & Coroner & $\begin{array}{l}\text { 1a Suicide by barbitone } \\
\text { poisoning }\end{array}$ & E950 & $\begin{array}{l}\text { 1a Barbitone poisoning by } \\
\text { sodium amityl. Open verdict }\end{array}$ & E980 \\
\hline .66116 & Hospital & $\begin{array}{l}\text { Ever- } \\
\text { user }\end{array}$ & No & Other & $\begin{array}{l}\text { 1a Subarachnoid haemorrhage } \\
\text { b Berry aneurysm } \\
\text { (Craniotomy four days before } \\
\text { death) }\end{array}$ & 430 & $\begin{array}{l}\text { 1a Cerebral infarction } \\
\text { b Spasm left middle cerebral } \\
\text { artery } \\
\text { c Aneurysm left cerebral artery }\end{array}$ & 442 \\
\hline 72859 & Hospital & Control & No & Other & $\begin{array}{l}\text { 1a Hepatic failure } \\
\text { b Infective hepatitis }\end{array}$ & 070 & $\begin{array}{l}\text { 1a Hepatic failure } \\
\text { b Acute hepatitis }\end{array}$ & 570 \\
\hline
\end{tabular}

- Denotes those deaths included in the RCGP publication of October 1977

The coded cause of death is shown in italics. 
occurred in 23 cases $(74 \%)$ and there were discrepancies in eight cases $(26 \%)$; the details of these are also listed in Table 2.

In Table 3 non-violent deaths are analysed in respect of the availability of post-mortem information, the certifier of death, and the place of death. A comparison is made between the coding at the ' $B$ ' list level, in which there was agreement, and those codings which did not agree. Not surprisingly, there is good agreement in cases where the GPs completed the death certificates, since they also provided the information for the study. Discrepancies are most likely to occur when a necropsy is performed, when a coroner completes the death certificate, or when a death occurs at home.

\section{(b) Circulatory diseases}

There were 57 deaths coded as due to circulatory diseases by either the RCGP or the registries, or both. These were compared by the ' $A$ ' list categories. Table 4 shows that in 48 cases (84\%) the RCGP

Table 3 Distribution of post-mortem information, certifier of death, and place of death in non-violent deaths by comparison of RCGP and OPCS/GRO coding at 'B' list level

\begin{tabular}{|c|c|c|c|c|c|}
\hline & $\underset{\%}{A G R E E D}$ & (No.) & & $\begin{array}{l}E D D \\
(N o .)\end{array}$ & $\begin{array}{l}\text { Total no. } \\
(=100 \%)\end{array}$ \\
\hline $\begin{array}{l}\text { (a) NECROPSY } \\
\text { Performed } \\
\text { Not performed }\end{array}$ & $\begin{array}{l}81 \\
91\end{array}$ & $\begin{array}{r}(44) \\
(106)\end{array}$ & $\begin{array}{r}19 \\
9\end{array}$ & $\begin{array}{l}(10) \\
(11)\end{array}$ & $\begin{array}{r}54 \\
117\end{array}$ \\
\hline $\begin{array}{l}\text { (b) CERTIFIER } \\
\text { OF DEATH }\end{array}$ & & & & & \\
\hline $\begin{array}{l}\text { Coroner } \\
\text { GP } \\
\text { Other }\end{array}$ & $\begin{array}{l}78 \\
96 \\
89\end{array}$ & $\begin{array}{r}(25) \\
(22) \\
(103)\end{array}$ & $\begin{array}{r}22 \\
4 \\
11\end{array}$ & $\begin{array}{r}(7) \\
(1) \\
(13)\end{array}$ & $\begin{array}{r}32 \\
23 \\
116\end{array}$ \\
\hline $\begin{array}{l}\text { (c) PLACE OF } \\
\text { DEATH }\end{array}$ & & & & & \\
\hline $\begin{array}{l}\text { Home } \\
\text { Hospital } \\
\text { Not known }\end{array}$ & $\begin{array}{l}89 \\
84 \\
97\end{array}$ & $\begin{array}{l}(33) \\
(81) \\
(36)\end{array}$ & $\begin{array}{r}11 \\
16 \\
2\end{array}$ & $\begin{array}{r}(4) \\
(16) \\
(1)\end{array}$ & $\begin{array}{l}37 \\
97 \\
37\end{array}$ \\
\hline
\end{tabular}

codes agreed entirely with the registry codes. There were nine discrepancies (16\%), the details of which are listed in Table 2. There was no systematic difference between the RCGP and the registry codes. In four cases $(7 \%)$ the RCGP coded a circulatory disease as the underlying cause of death, whereas the registries recorded a cause of death other than circulatory disease, and in three cases (5\%) the registries coded circulatory disease as the underlying cause of death, whereas the RCGP recorded some other cause.

\section{(c) Comparison of ever-users and controls}

Comparison of deaths in ever-users (102) with deaths in controls (69) by non-violent causes showed that 71 cases $(70 \%)$ and 51 cases $(74 \%)$ respectively agreed at the three-digit level. Eighty-eight cases $(86 \%)$ and 55 cases $(80 \%)$ respectively agreed in the ' $A$ ' list and $89 \%$ and $86 \%$ ( 91 and 59 cases respectively) agreed in the ' $B$ ' list. Codings in $11 \%$ and $14 \%$ ( 11 and 10 cases respectively) were discrepant in the ' $\mathrm{B}$ ' list. It is apparent, therefore, that discrepancies were no more likely to occur in ever-users than in non-users. Tables $5 \mathrm{a}$ and $5 \mathrm{~b}$ show that there was no systematic difference between RCGP and registry coding in ever-users and controls, which is especially important in the case of circulatory deaths.

DEATHS INCLUDED IN PUBLICATION OF OCTOBER 1977

Ninety-nine deaths were analysed in October 1977. Two deaths from complications of pregnancy were excluded at that time, together with 17 deaths from causes diagnosed before recruitment to the study. Table 6 shows the distribution of the 99 deaths by broad $I C D$ groups according to the original coding by the RCGP (as tabulated in the October 1977 publication) and the registry codes on the death certificates. Death certificates were unobtainable in 15 cases. Full details of discrepant cases within the

Table 4 Comparison of deaths attributed to circulatory diseases by RCGP or OPCS/GRO, by 'A' list ICD codes

\begin{tabular}{|c|c|c|c|c|c|c|c|c|c|}
\hline \multicolumn{10}{|c|}{ RCGP STUDY } \\
\hline & & $\begin{array}{l}\text { A82 } \\
\text { (Hypertensive } \\
\text { disease) }\end{array}$ & $\begin{array}{l}\text { A83 } \\
\text { (Ischaemic } \\
\text { heart } \\
\text { disease) }\end{array}$ & $\begin{array}{l}\text { A84 } \\
\text { (Other forms } \\
\text { of heart } \\
\text { disease) }\end{array}$ & $\begin{array}{l}\text { A85 } \\
\text { (Cerebro- } \\
\text { vascular } \\
\text { disease) }\end{array}$ & $\begin{array}{l}\text { A86 } \\
\text { (Disease of } \\
\text { arteries, } \\
\text { arterioles and } \\
\text { capillaries) }\end{array}$ & $\begin{array}{l}\text { A87 } \\
\text { (Venous } \\
\text { thrombosis } \\
\text { and embolism) }\end{array}$ & Other & Total \\
\hline OPCS/GRO & $\begin{array}{l}\text { A82 } \\
\text { A83 } \\
\text { A84 } \\
\text { A85 } \\
\text { A86 } \\
\text { A87 } \\
\text { Other }\end{array}$ & $\frac{1}{1}$ & 15 & 3 & $\frac{25}{1^{\circ}}$ & $\frac{1}{1^{*}}$ & 3 & $\begin{array}{l}1^{*} \\
1^{*} \\
1^{*}\end{array}$ & $\begin{array}{r}1 \\
17 \\
3 \\
26 \\
2 \\
4 \\
4\end{array}$ \\
\hline & Total & 2 & 15 & 3 & 29 & 2 & 3 & 3 & 57 \\
\hline
\end{tabular}

Patient numbers 10695, 14290, 16328, 17565, 35538, 33967, 42479, 61015, 66116 (see Table 2 for details). 
specified groups between RCGP and registry codes are listed and asterisked in Table 2. It is apparent that the distribution of underlying causes of death is little affected according to whether RCGP or registry codes are used for analysis.

\section{Discussion}

There is good agreement (72\%) between the RCGP and the registry death certificate at the three-digit level in the analyses of total deaths. Examination of the ' $B$ ' list discrepancies suggests that coding was usually compatible with the information given, although there are some errors. In 10 cases, similar wording was available to the registries and the RCGP. These were carefully reconsidered by the

Table 5 Comparison of deaths in ever-users and controls as coded by RCGP or OPCS/GRO with special reference to circulatory deaths (ICD 390-458)

\begin{tabular}{|c|c|c|c|c|c|}
\hline \multirow[t]{2}{*}{ (a) EVER- } & \multicolumn{3}{|c|}{$R C G P$} & \multirow[b]{2}{*}{ Total } & \multirow[b]{2}{*}{$\%$} \\
\hline & $\begin{array}{l}\text { Circulatory } \\
\text { deaths }\end{array}$ & $\begin{array}{l}\text { Violent } \\
\text { deaths }\end{array}$ & $\begin{array}{l}\text { Other } \\
\text { deaths }\end{array}$ & & \\
\hline \multicolumn{6}{|l|}{ OPCS/GRO } \\
\hline $\begin{array}{l}\text { Circulatory } \\
\text { deaths }\end{array}$ & & $\mathbf{0}$ & $2 *$ & 43 & 35 \\
\hline $\begin{array}{l}\text { Violent } \\
\text { deaths }\end{array}$ & $1 * *$ & & 0 & 22 & 18 \\
\hline $\begin{array}{l}\text { Other } \\
\text { deaths }\end{array}$ & $1^{* * *}$ & 0 & 20 & 59 & 47 \\
\hline Total & 43 & 21 & 60 & 124 & 100 \\
\hline$\%$ & 35 & 17 & 48 & 100 & \\
\hline
\end{tabular}

-Patient numbers 17565, 35538

**Patient number 16328

***Patient number 14290

See Table 2 for details.

(b) CONTROLS

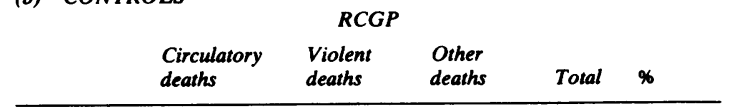

\begin{tabular}{|c|c|c|c|c|c|}
\hline \multicolumn{6}{|l|}{ OPCS/GRO } \\
\hline $\begin{array}{l}\text { Circulatory } \\
\text { deaths }\end{array}$ & & 0 & $1^{*}$ & 10 & 12 \\
\hline $\begin{array}{l}\text { Violent } \\
\text { deaths }\end{array}$ & 0 & & $2^{* *}$ & 12 & 15 \\
\hline $\begin{array}{l}\text { Other } \\
\text { deaths }\end{array}$ & $2^{* * *}$ & 0 & & 59 & 73 \\
\hline Total & 11 & 10 & 60 & 81 & 100 \\
\hline$\%$ & 14 & 12 & 74 & 100 & \\
\hline
\end{tabular}

-Patient number 61015

*Patient numbers 15595,30279

*** Patient numbers 33967, 42479

See Table 2 for details. registries. They concluded that, based on $I C D$ rules alone, two had been miscoded by them and eight by the RCGP. The discrepancies are not surprising, since the registries used international rules to assign underlying cause of death, and the regulations are on occasion complex and may even seem contrary to medical knowledge. On the other hand, in assigning the cause of death the RCGP sometimes used information relating to the previous medical history which did not appear on the death certificate. For example, patient number 17920 who had a past history of diabetes mellitus and asthma was reported to the RCGP to have been admitted to hospital four months before death with acute asthma, which was, therefore, coded as the underlying cause of death. The death certificate listed: 1a Bronchial asthma; $1 \mathrm{~b}$ Diabetes mellitus, and because of ICD coding regulations the latter was coded as the underlying cause of death by the registries. In this case the decision by the RCGP about the most important underlying cause of death was influenced by information not available on the death certificate. However, among the 205 deaths, such instances were uncommon.

Table 3 shows that a post-mortem examination was more likely to have been carried out in discrepant cases than in cases where coding agreed. An important reason for this is that post-mortem examinations are uncommon when the cause of death is obvious. For example, only $14 \%$ of women with cancer recorded either by the RCGP or by the registries had a necropsy, and yet there was $98 \%$ agreement on the underlying cause of death. Furthermore, post-mortem findings were not necessarily known to the GP when reporting on the cause of death, but this applied only to a small proportion of cases. Certain discrepancies appear to arise from subtleties of interpretation. For example, it is sometimes difficult to know whether the cause of death is accidental or not. In our study there are three cases (patient numbers 15595,16328 , and 30279) where the death was coded as non-violent by the RCGP and violent by the registries, although all three had been coded correctly from the information supplied. In all three cases post-mortem information was available. Patient 16328 , who had a massive cerebral haemorrhage associated with a fall at work, illustrates that even at inquest it was not possible to ascertain which of the two factors was the underlying cause of death. However, such instances are so infrequent that they are unlikely to disturb the outcome of epidemiological analyses, but they could account for the higher percentage of discrepancies observed when coroners complete the death certificates, since they do not routinely inform the GP of their conclusions. 
Table 6 Distribution of deaths analysed in October 1977 based upon original coding by the RCGP and OPCS/GRO codes on death certificates

\begin{tabular}{|c|c|c|c|c|c|}
\hline \multirow[b]{2}{*}{ CAUSE OF DEATH } & \multirow[b]{2}{*}{$I C D$} & \multicolumn{2}{|c|}{ (a) Original coding by RCGP* } & \multicolumn{2}{|c|}{ (b) OPCS/GRO death certificates } \\
\hline & & EVER-USERS & CONTROLS & EVER-USERS & CONTROLS \\
\hline All cancers & $140-209$ & 12 & 20 & 11 & 20 \\
\hline $\begin{array}{l}\text { All diseases of the } \\
\text { circulatory system }\end{array}$ & $\begin{array}{l}390-458 \\
400-429 \\
430-438\end{array}$ & $\begin{array}{r}21 \\
10 \\
9\end{array}$ & $\begin{array}{l}3 \\
2 \\
2\end{array}$ & $\begin{array}{r}20 \\
9 \\
8\end{array}$ & $\begin{array}{l}2 \\
2 \\
0\end{array}$ \\
\hline $\begin{array}{l}\text { Accidents and } \\
\text { violence }\end{array}$ & $\begin{array}{c}\text { E800-999 } \\
\text { E800-949 } \\
\text { E980 } \\
\text { E950-999 } \\
\text { E960-999 }\end{array}$ & $\begin{array}{l}12 \\
7 \\
4 \\
1\end{array}$ & $\begin{array}{l}7 \\
6 \\
1 \\
0\end{array}$ & $\begin{array}{r}12 \\
9 \\
3 \\
0\end{array}$ & $\begin{array}{l}8 \\
7 \\
1 \\
0\end{array}$ \\
\hline Other causes & & 2 & 7 & 4 & 7 \\
\hline $\begin{array}{l}\text { Death certificate } \\
\text { unobtainable, there } \\
\text { unmatched }\end{array}$ & & 9 & 6 & 9 & 6 \\
\hline TOTAL & & 56 & 43 & 56 & 43 \\
\hline
\end{tabular}

- Because of the distribution of unobtainable death certificates, the numbers in section (a) are not identical to those which appear in Table 1 of the RCGP publication in October 1977.

There was no evidence of bias in terms of circulatory disease, nor of oral contraceptive use or non-use. It should be emphasised that the occasional errors in the RCGP codings of deaths not previously analysed would have been identified and corrected at the second stage of validation.

Table 5 shows that the distribution of deaths analysed in October 1977 according to registry coding is very similar to the distribution reported with RCGP codes in both ever-users and controls. There is no reason why the unobtainable death certificates should be biased, and it seems reasonable to conclude that the previously estimated mortality risks associated with oral contraceptive usage would not be materially altered by use of death certificate information. This study, of course, is not a direct test of the accuracy of death certificates, because in 25 patients $(10 \%$ of cases) the GPs themselves completed both the death certificate and the forms for the RCGP study. On the other hand, the coding of the underlying cause of death was independent.

There has been much debate on the inaccuracies of death certificates ${ }^{587}$ and their validity for the purposes of epidemiological research. Many variables are involved in arriving at any final code of death, namely certifier, available information, such as previous medical history and post-mortem report, and, finally, the coding regulations employed. The underlying cause of death assigned by the RCGP may represent a more realistic assessment than that coded from the death certificate, by virtue of the availability and use of previous medical history. Nevertheless, the RCGP coding method, essentially a modification of ICD recommendations, would be neither practicable nor appropriate on a national scale. Where trained coders, rather than doctors, are involved, the systematic rules of the ICD are successful. Weaknesses in both systems do exist, and serve to demonstrate that perfection is impossible, even where post-mortem findings are available. In general, the errors described here and in other studies of cause of death certification only mirror what happens in clinical medicine. In certain subtle situations there is inevitably a choice of underlying cause. The assignment of the 'cause' may vary from one clinician to another and at different times, but the power of cause of death certification in research has repeatedly been demonstrated. ${ }^{78}$ The strong agreement between the ' $B$ ' list codes assigned by the RCGP and by the registries observed in this study is evidence of the effectiveness of both methods for analyses of the study data, and probably also for other epidemiological purposes.

We thank the 1400 GPs who are contributing all the data for this survey. The study is supported by a major grant from the Medical Research Council. The costs of the pilot trials and current supplementary expenditure have been met by the Scientific 
Foundation Board of the Royal College of General Practitioners. The board gratefully acknowledges the receipt of funds for research into oral contraception from Organon Laboratories Ltd., Ortho Pharmaceutical Corporation, Schering Chemicals Ltd., G. D. Searle and Co. Ltd., Syntex Pharmaceuticals Ltd., and John Wyeth and Brother Ltd.

Reprints from Dr. Clifford R. Kay, Royal College of General Practitioners, Manchester Research Unit, 8 Barlow Moor Road, Manchester M20 0TR.

\section{References}

${ }^{1}$ Royal College of General Practitioners Oral Contraception Study. Mortality among oral contraceptive users. Lancet 1977; ii: 727-31.
${ }^{2}$ Royal College of General Practitioners. Oral Contraceptives and Health. London: Pitman Medical, 1974.

${ }^{3}$ World Health Organisation. Manual of the international statistical classification of diseases, injuries, and causes of death. 8th revision, 1965. Geneva: WHO, 1967.

${ }^{4}$ Reid DD, Rose GA. Assessing the comparability of mortality statistics. Br Med J 1964; ii: 1437-9.

${ }^{5}$ Medical Services Study Group of the Royal College of Physicians of London. Death certification and epidemiological research. $\mathrm{Br}$ Med J 1978; ii: 1063-5.

${ }^{6}$ Clarke C, Whitfield AGW. Deaths from rhesus haemolytic diseases in England and Wales in 1977: Accuracy of records and assessment of anti-D prophylaxis. $\mathrm{Br} \mathrm{Med} J \mathrm{1979}$; i: $1665-9$.

${ }^{7}$ Adelstein AM. Death certificates and epidemiological research. Br Med J 1978; ii: 1229-30.

${ }^{8}$ Adelstein AM. Current vital statistics: methods and interpretations. $\mathrm{Br}$ Med J 1978; ii: 983-7. 